EXTENDED REPORT

\title{
A naturalistic study of the determinants of health related quality of life improvement in osteoarthritic patients treated with non-specific non-steroidal anti- inflammatory drugs
}

\author{
V Rabenda, N Burlet, O Ethgen, F Raeman, J Belaiche, J-Y Reginster
}

Ann Rheum Dis 2005;64:688-693. doi: 10.1136/ard.2004.026658

See end of article for authors' affiliations

Correspondence to: Mrs V Rabenda, Service de Santé Publique,

Epidémiologie et Economie de la Santé, 3 avenue de l'Hôpital, Bâtiment 23,

CHU Sart-Tilman, 4000

Liège, Belgium;

veronique.rabenda@

ulg.ac.be

Accepted 9 October 2004

Published Online First

4 November 2004

\begin{abstract}
Objectives: To capture changes in the quality of life (QoL) occurring in patients with osteoarthritis (OA) during treatment with non-specific non-steroidal anti-inflammatory drugs (NSAIDs) and to identify factors that predict such changes.

Methods: A naturalistic, prospective follow up of 783 patients with $O A$ in whom primary care physicians decided to start treatment with non-selective NSAIDs. Short Form-36 (SF-36) and the Western Ontario and McMaster Universities OA index (WOMAC) were assessed at baseline and after 3 months. Baseline results were compared with QoL values in 4800 subjects randomly selected from the general population. Multiple regression analysis was performed to identify determinants of QoL at baseline and measures influencing changes in SF-36 or WOMAC during follow up.

Results: All QoL dimensions were significantly $(\mathrm{p}<0.01)$ decreased in patients with $O A$ compared with controls. Significant improvement $(p<0.05)$ in four dimensions of the SF-36 (vitality, role emotional, role physical, bodily pain) and in all components of the WOMAC was seen between baseline and month 3 . Older age, female sex, longer duration of OA, and a higher number of comorbidities were the major determinants of a poor QoL at baseline. Maximal benefit from non-specific NSAIDs was seen in patients with the most severe impairment in QoL and the shortest duration of OA.

Conclusion: OA negatively impacts all dimensions of the QoL. Non-specific NSAIDs improve the QoL in patients with OA treated in a "real life setting". The profile of patients receiving maximal benefit from such treatment may be of interest for health providers, enabling them to decide who should preferentially be given cytoprotective treatments or coxibs.
\end{abstract}

O teoarthritis (OA) is a chronic degenerative joint disease and a major source of disability in elderly people. ${ }^{1}$ Disability may be characterised as the impaired performance of expected socially defined life tasks, in a typical sociocultural and physical environment. ${ }^{2}$ Elderly people with physical and psychosocial disability have been repeatedly shown to have lower levels of health related quality of life (QoL) than matched controls, ${ }^{45}$ a condition also associated with the presence of various degrees of OA. ${ }^{67}$ Health related QoL outcomes provide an effective means for clinicians to make clinically sensible decisions by providing further insight into the benefits and drawbacks of treatment options. $^{7}$

Although there are few doubts, today, that arthritic conditions may significantly alter the QoL, particularly in elderly people, ${ }^{67}$ there is still some debate about the relative impact of OA on the different dimensions of QoL, ${ }^{8}$ the respective usefulness of generic or specific instruments to measure QoL in OA, ${ }^{6}$ the determinants of disability or QoL changes over time, ${ }^{2}$ and the impact of treatments for OA on QoL. $^{79}$

Symptomatic treatments of OA consist of non-pharmacological as well as pharmacological interventions, including the use of non-steroidal anti-inflammatory drugs (NSAIDs). NSAIDs reduce inflammation, alleviate pain, and maintain functional activity. However, conventional (non-selective) NSAIDs may induce upper gastrointestinal (GI) side effects, which may, in turn, negatively impact the QoL. ${ }^{10}$

We designed the present naturalistic trial to assess whether patients starting treatment with a conventional
NSAID for the symptomatic management of OA had an impaired QoL. We prospectively followed up the cohort to capture changes in QoL over time and to identify the main determinants of such changes. We compared the information obtained from generic and specific health related QoL instruments.

\section{METHODS}

This study is an ancillary protocol to a naturalistic, prospective survey, designed with the primary objective of evaluating gastroprotective drug (GPD) co-prescriptions in patients treated with NSAIDs by general practitioners (GPs). Fifty primary care physicians-that is, Belgian GPs, were asked to recruit 50 consecutive patients each, for whom they felt it was appropriate, based on their normal practice, to start treatment with non-selective NSAIDs. Data were collected between August 2001 and December 2002. Cyclo-oxygenase-2 inhibitors (coxibs) were not marketed and reimbursed in Belgium before September 2002. Men and women aged over 35 years were included. One hundred patients were aged between 35 and 50 and 683 patients were older than 50 . The only exclusion criteria were previous exposure to investigational coxibs at any time, exposure to NSAIDs

Abbreviations: coxibs, cyclo-oxygenase-2 inhibitors; $\mathrm{Gl}$, gastrointestinal; GPD, gastroprotective drug; GPs, general practitioners; NSAIDs, non-steroidal anti-inflammatory drugs; OA, osteoarthritis; QoL, quality of life; RCTs, randomised controlled trials; SF-36, Short Form-36; WOMAC, Western Ontario and McMaster Universities Osteoarthritis Index 
Table 1 Type, dose, and duration of the five NSAIDs most used during the study period

\begin{tabular}{llcclll}
\hline NSAIDs & $\mathbf{n}$ & $\begin{array}{l}\text { Mean daily } \\
\text { dose }(\mathbf{m g})\end{array}$ & $\mathbf{9 5 \%} \mathrm{Cl}$ & $\begin{array}{l}\text { Less than } \\
\mathbf{1} \text { week } \\
\text { (n) }\end{array}$ & $\begin{array}{l}\mathbf{1 - 4} \text { Weeks } \\
\text { (n) }\end{array}$ & $\begin{array}{l}1-3 \text { Months } \\
\text { (n) }\end{array}$ \\
\hline Piroxicam & 232 & 20.04 & 19.69 to 20.39 & 113 & 24 & 81 \\
Nimesulide & 117 & 178.88 & 170.14 to 187.62 & 14 & 58 & 41 \\
Meloxicam & 100 & 19.32 & 13.55 to 25.09 & 8 & 46 & 43 \\
lbuprofen & 129 & 1055.2 & 975.41 to 1134.99 & 16 & 72 & 36 \\
Diclofenac & 118 & 120.99 & 114.02 to 127.96 & 8 & 62 & 46 \\
\hline
\end{tabular}

during the past 3 months, and concomitant use of corticosteroids, anticoagulants, or low dose aspirin. GPs were instructed to prescribe NSAIDs (type, dose, and duration) according to their usual standard of care. To speed up recruitment, the number of GPs participating in the survey was increased to 66.

Patients were divided into three diagnostic groups, based on the underlying disease leading to the NSAID prescription: OA, chronic back pain without OA, and any other medical condition. At baseline, we collected information on a previous history of GI disorders and GPD use during the past 3 months. Patients were also asked to report spontaneously any chronic and/or severe disorders they had had during the previous 6 months. A list of chronic conditions was then presented to the participants in order to exclude any forgotten disease. Patients were seen by their GPs after 3 months and information was collected on NSAID intake (persistence), occurrence of GI adverse reactions, and GPD prescriptions (nature and motivation).

At baseline and after 3 months, patients from the OA group were asked to complete two health related QoL instruments: the Medical Outcomes Study Short Form-36 (SF-36) and the Western Ontario and McMaster Universities OA Index (WOMAC).

The SF-36 is a generic health related QoL instrument which consists of 36 items that measure eight dimensions of health status as reported by the patient. Four dimensions refer to physical health: limitations in physical functioning due to health, limitations in usual role activities due to physical health, bodily pain, and perceptions of health in general. Four dimensions allude to mental health: vitality (energy and fatigue), limitations in social activities due to physical or emotional health, limitations in usual role activities due to personal or emotional problems, and general mental health (psychological distress and wellbeing). ${ }^{11}{ }^{12}$ These dimensions are scored from 0 to 100, with lower scores indicating lower levels of health related QoL. One of the strongest attributes of the SF-36 is its consistently high levels of reliability (test-retest and internal consistency) and validity (content, concurrent, criterion, construct, and predictive). ${ }^{12}{ }^{13}$ The SF-36 has been broadly applied and validated for the measurement of health outcomes in diverse languages (including French ${ }^{14}{ }^{15}$ ) and for various conditions (including OA).

Besides this generic instrument, we also used the WOMAC, which is a validated and self reported health status instrument for measurement of the symptoms of OA of the hips and knees. The WOMAC separately deals with severity of joint pain (5 questions), stiffness ( 2 questions), and limitation of physical function ( 17 questions) in the 48 hours before assessment. The Likert scale version of the WOMAC index was used, with each question scored on a scale from 0 to 4 , with 0 indicating none and 4 indicating extreme; 20, 8 , and 68 points, therefore, are the worst possible severity scores of pain, stiffness, and limitation of physical function, respectively. ${ }^{16}$

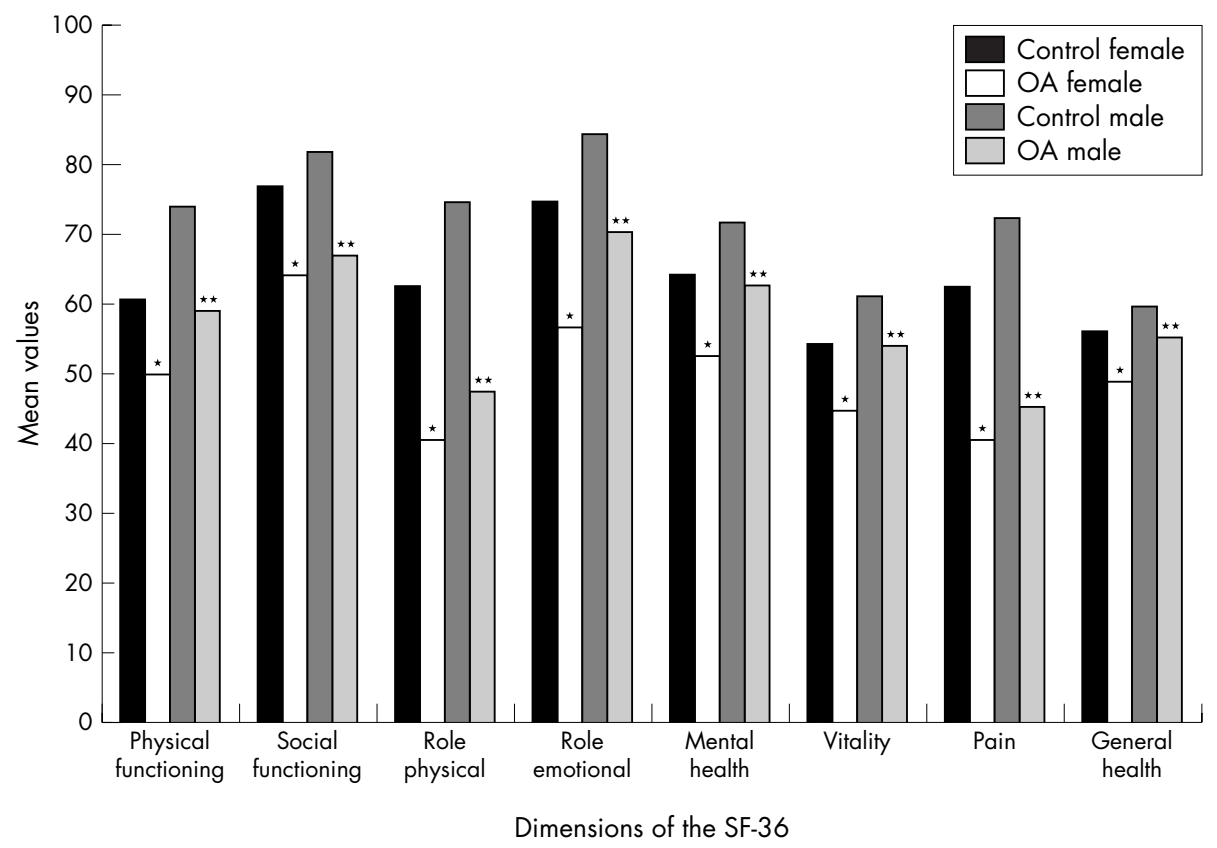

Figure 1 Mean values of the various dimensions of the SF-36, by sex, in 783 subjects with OA and in a control cohort obtained from a random sample of 4800 Belgian citizens. 
Table 2 Mean values of the total WOMAC index and its subscales for the entire OA group, men, and women, at baseline and after 3 months of follow up

\begin{tabular}{|c|c|c|c|c|c|c|}
\hline & \multicolumn{2}{|l|}{ Men } & \multicolumn{2}{|l|}{ Women } & \multicolumn{2}{|l|}{ All } \\
\hline & Baseline & Month 3 & Baseline & Month 3 & Baseline & Month 3 \\
\hline Pain & 6.99 & 6.23 & 8.72 & 7.63 & 8.2 & $7.19^{*}$ \\
\hline Stiffness & 3.61 & 3.33 & 4.09 & 3.78 & 3.95 & $3.65^{*}$ \\
\hline Physical & 26.2 & 24.06 & 31.92 & 29.23 & 30.2 & $27.62^{*}$ \\
\hline Total & 36.8 & 33.62 & 44.75 & 40.66 & 42.37 & $38.47^{*}$ \\
\hline
\end{tabular}

Owing to the naturalistic approach of our study, no direct control, using either a healthy population or an untreated OA population, was possible. We compared the baseline values of the SF-36 with those found in a historical control-that is, a representative random sample of 4800 Belgian subjects, aged over 45 years, who had taken part in a population health survey organised by the Belgian National Social Security Institute (INAMI-RIZIV) and for which SF-36 data were available. We have exhaustively described the methodology of recruitment of this population sample in a previous publication. ${ }^{17}$ Matched subjects were selected to constitute a control group. The two matching criteria were age and sex. The whole database allowed us to recover three matched subjects for each patient with OA.

\section{Statistics}

An unpaired Student's $t$ test was used for comparison of the SF-36 values found in the OA group and in the historical controls. SF-36 or WOMAC values between inclusion and the month 3 visit were assessed by a paired Student's $t$ test. Multiple regression analysis was performed to identify the determinants of health related QoL at baseline in order to validate our population in comparison with previous studies and the measures influencing the changes in the WOMAC and SF-36 seen during the 3 months of the trial.

\section{RESULTS}

Seven hundred and eighty three patients with OA were included in the present trial. They had a mean (SD) age of 66.5 (12.5) years, and included $533(68.1 \%)$ women. They reported a mean number of 2.2 comorbidities. At baseline, the NSAIDs most frequently prescribed by the GPs were piroxicam $(n=205)$, ibuprofen $(n=109)$, diclofenac $(\mathrm{n}=112)$, nimesulide $(\mathrm{n}=93)$, and meloxicam $(\mathrm{n}=92)$. Altogether, these five most commonly prescribed NSAIDs totalled 611 (78\%) of the GPs prescriptions. Table 1 shows the type, mean daily dose, and duration of the five NSAIDs most commonly used during the study. Only 18 patients consulted a physical therapist during the 3 month follow up.

The SF-36 questionnaire was available for all patients at baseline and after 3 months. Valid data were obtained for all dimensions of the SF-36 in more than $94 \%$ at baseline and in more than $93 \%$ of the subjects after 3 months. At baseline and after 3 months $593(75.7 \%)$ and 586 (74.8\%) subjects filled the WOMAC questionnaire, respectively. Figure 1 shows the values of the various dimensions of the SF-36, by sex, at baseline, in our OA population and in the historical cohort. Values were significantly lower, for all dimensions and for both sexes in comparison with the historical controls (age 67.7 (11.0) years, percentage of women 68.4\%, number of comorbidities 1.9) (fig 1). When we adjusted for age and sex, all dimensions of the SF-36 were also significantly lower for patients with OA than for the historical controls.

Table 2 reports the baseline and 3 month values of the WOMAC in the OA population, for both sexes and for the total population. From baseline to the 3 month evaluation, a significant improvement $(\mathrm{p}<0.05)$ was observed for the domains of vitality, role physical, role emotional, and bodily pain (fig 2). The values of the total WOMAC index and each of the subscales were significantly $(\mathrm{p}<0.05)$ improved between baseline and month 3 (table 2 ).

For most dimensions of the SF-36 (except social functioning and physical role), and for all components of the WOMAC, the multiple regression analysis identified sex of

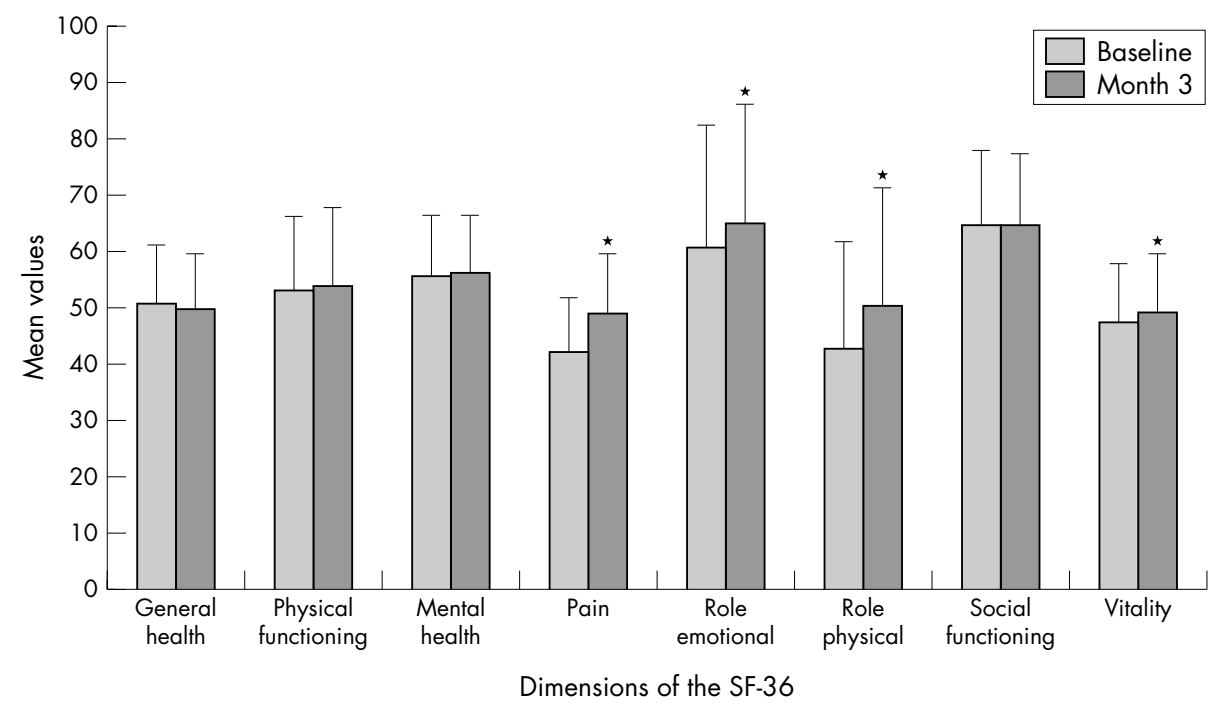

Figure 2 Mean values of the various dimensions of the SF-36 at baseline (>94\% of the population) and at 3 months (>93\% of the population) in a sample of 783 patients with OA. 
Table 3 Multiple regression summary. Determinants of change in the dimensions of the SF-36 between baseline and month 3

\begin{tabular}{lccclll}
\hline & Intercept & Age & Sex & $\begin{array}{l}\text { Baseline } \\
\text { score }\end{array}$ & $\begin{array}{l}\text { Duration of } \\
\text { OA }\end{array}$ & $\begin{array}{l}\text { Number of } \\
\text { comorbidities at } \\
\text { baseline }\end{array}$ \\
\hline General health & $13.06^{*}$ & 0.007 & 0.74 & $-0.25^{*}$ & -0.08 & $-1.07^{*}$ \\
Physical functioning & $20.46^{*}$ & -0.09 & 2.05 & $-0.21^{*}$ & -0.14 & $-1.45^{*}$ \\
Mental health & $13.56^{*}$ & 0.05 & -0.78 & $-0.24^{*}$ & -0.09 & -0.49 \\
Pain & $29.89^{*}$ & -0.06 & 2.09 & $-0.37^{*}$ & $-0.23^{*}$ & $-1.6^{*}$ \\
Role emotional & $25.91^{*}$ & 0.005 & 1.95 & $-0.4^{*}$ & -0.02 & -1.57 \\
Role physical & $34.59^{*}$ & -0.05 & 3.26 & $-0.44^{*}$ & $-0.41^{*}$ & $-2.56^{*}$ \\
Social functioning & $26.32^{*}$ & -0.08 & 0.36 & $-0.31^{*}$ & -0.01 & -0.41 \\
Vitality & $-8.47^{*}$ & 0.008 & -0.31 & $0.76^{*}$ & $-0.17^{*}$ & -0.35 \\
\hline${ }^{*} p<0.05$. & & & & & & \\
\hline
\end{tabular}

the patient (men have a better QoL than women) as a significant and independent predictor of QoL at baseline. An increased prevalence of comorbidities is linked to a poorer QoL (SF-36 and WOMAC). For the SF-36 physical function and mental health dimensions, and for the function and total scales of the WOMAC, age (QoL decreasing in advanced age) is also a significant component of the QoL. The duration of $\mathrm{OA}$ is independently related to the QoL in the vitality dimension of the SF-36 (longer duration of OA decreases the QoL), but not in the WOMAC.

When assessing, by multiple regression analysis, the determinants of improvement in the QoL, in patients with OA treated with conventional NSAIDs, it was found that the patients with the lowest level of QoL at baseline (SF-36 and WOMAC) had the greatest improvement in QoL over time. A low number of comorbidities at baseline was associated with the greatest improvement in QoL (physical functioning, role physical, pain, and general health dimensions of the SF-36 and pain subscale of the WOMAC) (table 3 and 4). A shorter duration of disease (role physical, vitality, and pain in the SF36) was also a factor associated with a higher improvement in QoL after 3 months (table 3).

\section{DISCUSSION}

Several studies have previously reported that patients with OA, with or without comorbidities, are characterised by a poorer QoL than their peers, particularly in the domains associated with physical status, but also in those of vitality, social functioning, and general health. ${ }^{4}{ }^{8}$ Our results support these findings, with all dimensions of the SF-36 being altered in both men and women with OA, compared with a representative sample of the general population of similar age and comorbidities. ${ }^{17}$

We should acknowledge, however, two particular aspects of our OA cohort. These patients were subjects for whom primary care physicians identified the need to start treatment with NSAIDs. This strongly suggests that these patients were symptomatic at the time of assessment of the QoL. Because OA may be characterised by phases of flares and respite, the

Table 4 Multiple regression summary. Determinants of change in the dimensions of the WOMAC between baseline and month 3

\begin{tabular}{|c|c|c|c|c|c|c|}
\hline & Intercept & Age & Sex & $\begin{array}{l}\text { Baseline } \\
\text { score }\end{array}$ & $\begin{array}{l}\text { Duration } \\
\text { of OA }\end{array}$ & $\begin{array}{l}\text { Number of } \\
\text { comorbidities } \\
\text { at baseline }\end{array}$ \\
\hline Pain & 0.06 & 0.007 & -0.11 & $-0.26^{*}$ & 0.03 & $0.23^{*}$ \\
\hline Stiffness & 0.27 & 0.01 & -0.09 & $-0.34^{*}$ & 0.01 & 0.05 \\
\hline Physical & 0.63 & 0.03 & -0.59 & $-0.2^{*}$ & 0.04 & 0.44 \\
\hline Total & -0.83 & 0.04 & -0.4 & $-0.17^{*}$ & 0.07 & 0.59 \\
\hline
\end{tabular}

results we observed, particularly for the pain and the physical functioning dimensions, may be slightly overestimated compared with the whole population of patients with OA.

Owing to the naturalistic design of our study, we did not ask the primary care physicians to perform any additional investigation to confirm the diagnosis of OA, in addition to their usual standard of care. We thus cannot exclude the possibility that some patients were mistakenly included in this trial and actually had other musculoskeletal conditions.

The values of the total WOMAC score obtained at baseline in our OA population reflect a slightly poorer condition than the one reported following a similar methodology, in a cohort of similar age (62.4 years) and percentage of women $(77.7 \%)$ in a trial designed to assess the efficacy of a potential structure modifying agent in knee $\mathrm{OA}^{18}$ In that particular study, $x$ ray examinations were performed at the time of the QoL assessment and showed a population equally distributed between Kellgren-Lawrence grades 2 and 3. This suggests that the severity of OA in our cohort could be considered as moderate to severe. Furthermore, the WOMAC has been designed to capture the essential elements of pain, stiffness, and physical functioning in patients with OA of the knee and/ or hip joints and because no skeletal radiography was systematically performed, we cannot rule out the possibility that our population included patients with OA at other locations than the lower limbs. Moreover, subjects presenting $\mathrm{OA}$ in the upper limbs did not answer this questionnaire, which explains the weaker effective size for the WOMAC compared with the SF-36.

The five most commonly prescribed NSAIDs represented $78 \%$ of the total number of prescriptions, in accordance with the national prescribing pattern of NSAIDs for GPs and also in agreement with previous publications performed in similar settings. ${ }^{19}$ In this naturalistic study, we did not ask the patient to complete diaries to record accurately the compliance rates for the NSAIDs. As previously published, such measures may have a significant impact on the degree of adherence and persistence of the patient to a drug and this would have jeopardised the naturalistic character of our study.

In absence of a control group, reflecting the natural history of the disease, our observation of a statistically significant improvement, over 3 months of treatment with conventional NSAIDs, in the domains of vitality, role physical, role emotional, and bodily pain evaluated by the SF-36 and in all dimensions of the WOMAC, remains of limited value. However, the magnitude of the improvement seen in our study after 3 months for the dimensions linked to pain (15$25 \%$ ) is within the same range as that reported in randomised clinical trials assessing, in OA of the lower limbs, the symptomatic efficacy of diclofenac, or meloxicam, two of the most commonly prescribed NSAIDs in our sample..$^{21}$ 
In this randomised controlled trial, however, the placebo effect in the OA population is reckoned to be also about $20 \%$. There are many explanations of this apparently contradictory result. Firstly, the "real life" design of our study did not allow for the inclusion of an untreated control population. Thus we cannot exclude the possibility that patients had a placebo effect and that the improvement observed for most of the dimensions of the SF-36 and for all dimensions of the WOMAC can be explained by a "doctor effect" rather than by the NSAID itself. Furthermore, outcomes in randomised controlled trials (RCTs) have been repeatedly shown to be influenced by the design of the study and to be rather different from those seen in daily practice. ${ }^{6822}$ The populations being treated in RCTs are usually highly selected groups and therefore are not always fully representative of the patients seen in clinical practice. In RCTs, the study group is as homogeneous as possible because patients with other possible rheumatic disorders are excluded. Moreover, patients who are included in RCTs are usually recruited on the basis of a minimal level of pain above average. The patterns of prescription, follow up, and data collection are far from the standards usually achieved in RCTs. For all these reasons, the results obtained in RCTs are usually greater than those obtained in "real life" settings.

Another question that could be asked about our results is whether the WOMAC is the appropriate tool for investigating pain in patients living in the "real life" setting. From our data and the observation that a certain proportion of patients $(20 \%)$ were not able to complete the WOMAC, we might reach the conclusion that the WOMAC is more appropriate for clinical trials than for naturalistic studies. As previously mentioned, we should, however, keep in mind that the WOMAC has been specifically designed for the assessment of pain in patients with OA of the lower limbs and is not fully validated for OA in other skeletal locations. In our study, the exact location of OA was not provided by the investigators (GPs), which may also explain the discrepancy between our results and those of previous clinical studies performed in OA of the lower limbs.

NSAID treatment has a marked impact on health related QoL. Not only pain and stiffness decreased but also the ability to perform routine tasks improved. Furthermore, in these patients the vitality and role emotional domains also seemed to improve, which might be because of their increased ability to perform and enjoy routine tasks and leisure activities while experiencing relief from the signs and symptoms of OA.

However, the data concerning the improvement in pain confirm that the patients did not return to integrity. Figures 1 and 2 show that the differences for SF-36 in the pain domain ranged from around 42 in the OA group at baseline to 49 after 3 months of treatment, whereas the control group scored about 67. Nevertheless, it is necessary to remain cautious in analysis of the results because we do not have a direct comparison with a control group. Furthermore, most of the RCTs which tested NSAIDs for the improvement in pain did not show full pain relief but only a statistically significant improvement. Bradley et al showed that a low dose of ibuprofen produces a $30 \%$ reduction in the pain caused by OA. ${ }^{23}$ Three months is not always sufficient time to allow the maximal reduction of pain to be reached.

Lin et al report a further meta-analysis exploring the use of topical NSAIDs in the treatment of OA. This well conducted study found that topical NSAIDs were better than placebo in reducing pain and improving function over 2 weeks, but that these effects were lost 4 weeks later. ${ }^{24}$ LaMontagna et al found that improvement in pain and functional capacity with two different NSAIDs was limited to the first few weeks of treatment; these measures then returned to baseline levels. These authors suggested that the treatment period in future trials with NSAIDs should not exceed 3 months because there seemed to be little clinical improvement in pain and function after this period. ${ }^{25}$ In our study, the OA localisation was not assessed, and QoL levels are known to be related to the site of the OA. This lack of information may explain the weak pain decrease after 3 months of follow up.

We previously reported a statistically significant improvement in four dimensions of the SF-36, but of relatively marginal magnitude (fig 2). It is generally believed that small differences in health related QoL may be statistically significant yet clinically irrelevant. The concept of the minimal clinically important difference (MCID) has been proposed to refer to the smallest difference in a score that is considered to be meaningful or clinically important. It can be defined generally as the smallest difference in score that the patients perceive as beneficial and which would then mandate a change in the patient's management. Reports of the SF-36 Health Survey shows that very small differences in the SF-36 could be interpreted as clinically important. ${ }^{26}$ Samsa et al concluded that the MCID for the Medical Outcomes Study Short Form-36 (SF-36) Health Survey is typically in the range of 3-5 points. This statement implies that differences in SF-36 scores of 1-2 points are deemed irrelevant.

Our observation that QoL in OA is negatively impacted by being a woman, being older, having a great number of comorbidities, or a longer duration of the disease, is in accordance with previous cross sectional $^{6}$ or prospective ${ }^{2}$ studies searching for factors that predict a severe impact of OA on physical functioning or QoL. More novative, however, in our report is that the best potential responders to conventional NSAIDs, with the greatest effect on QoL, are patients with a recent onset of OA and with symptoms severely affecting their QoL at baseline. This finding may be due to regression to the mean. Patients with lower baseline scores, reflecting worsening of their health status, have a higher probability of increasing the score at the follow up than patients with higher baseline scores, and vice versa. In the extreme case, at the end of the scale, only one direction of an effect $\neq 0$ is possible: for the SF-36, improvement when the baseline score is 0 (ceiling effect) and worsening when the baseline score is 100 (floor effect). ${ }^{27} 28$

Although conflicting results have been obtained about the overall benefit of non-specific NSAIDs on the QoL in arthritic patients, mainly owing to the negative impact generated by their GI toxicity, ${ }^{10}$ a few studies have aimed at defining the profile of patients who might expect the greatest benefit from such treatment in OA. To the best of our knowledge, this study is the first examining this question in a naturalistic design. From the perspective of a rational use of health resources, however, it is critically important to target patients with the highest expected benefit/risk ratio. A high number of comorbidities at baseline is detrimental to improvement in the QoL seen during the treatment of OA with NSAIDs. This is particularly true when assessing QoL with the SF-36, known to lack specificity for OA. Treatment with NSAIDs targets pain and function, the major symptoms of OA, but probably does not have a positive effect on the clinical expression and the impact on QoL of most comorbidities. This is in accordance with our observation that the best responders to NSAIDs, are those patients with OA with the highest impact of $\mathrm{OA}$ on the QoL at baseline. NSAIDs improve OA symptoms (pain, function) and subsequently alleviate the impact of OA on the QoL. Recent onset of OA is also a positive factor for response to NSAIDs.

There is no contradiction between these two findingsnamely, that patients whose QoL is severely affected and those who have a recent onset of OA have a better response to NSAIDs. A poor relationship has been shown between the 
radiological severity of $\mathrm{OA}$ - that is, an indirect expression of the duration of the disease and the magnitude of OA symptoms, measured by the WOMAC. ${ }^{29}$ We previously reported, from the primary analysis of this study ${ }^{30}$ that GI side effects requiring a GPD co-prescription, were significantly more common in patients aged 55 and older. The observation that patients with a recent onset of OA but severe impact on their QoL are the best responders to non-specific NSAIDs, whereas elderly patients have a higher risk of adverse reactions, may be of interest when discussing with health providers which patients will benefit from cytoprotective drugs or coxibs.

\section{ACKNOWLEDGEMENTS}

The authors acknowledge the support of Dr Muriel Haim and Dr Sheldon Kong, Outcomes Research department, Merck \& Co, Whitehouse Station NJ USA, who contributed to the design of the trial and partially funded it.

\section{Authors' affiliations}

V Rabenda, N Burlet, O Ethgen, J-Y Reginster, Department of Epidemiology, Public Health and Health Economics, University of Liège, Liège-Belgium and World Health Organisation (WHO) Collaborating Centre for Public Health Aspects of Rheumatic Diseases, Liège, Belgium F Raeman, Department of Internal Medicine, AZ Jan Palfijn Hospital, Antwerp, Belgium

J Belaiche, Department of Gastroenterology, CHU Sart-Tilman, University of Liège, Liège, Belgium

\section{APPENDIX: GENERAL PRACTITIONERS PARTICIPATING IN THIS TRIAL}

Archies Thierry, Arts Eric, Bauval Marcel, Bernardin Michel Bodson Philippe, Bolly Fernand, Bonaventure Robert, Brands Yves, Clarysse Laurent, Collet Nathalie, Dalpont Mario, David Philippe, De Cock Marc, De Roeck Paul, Defour Thierry, Dirick Paul, Disteche Stéphane, Dore Giovanni, Embrechts Guy, Formato Vincent, Franck Béatrice, George Gilbert, Gerard Pierre, Halfort Guy, Hillewaere Bart, Joly Michel, Lennartz Philippe, Leysen Paul, Lhoutte Alain, Mellaers Marc, Mersh Laurent, Meurant Jean-Pierre, Michaux JeanPaul, Moes Jean, Monet Francis, Mottet Paul, Neuprez Henri, Op de Beeck Guido, Payen Etienne, Perikel Françoise, Raeymaeckers Rudy, Raskin Corinne, Ravanelle Dominique, Rixhon Martine, Schroyen Marc, Sierens Geert, Simoens Ludo, Soumoy Norbert, Stockbroeckx Kris, Taziaux Marc, Taziaux Pierre, Thirion Michel, Thirion Pierre, Tuttino AnneMarie, van Braeckel Luc, van de Heijning Kurt, van Dijck Marc, van Look Carine, Vandeweerdt Jan, Verdin André, Wauthy Daniel, Wiame Pascal, Willekens Eric, Wuyard Alfred.

\section{REFERENCES}

1 Reginster J-Y. The prevalence and burden of arthritis. Rheumatology (Oxford) 2002;41(suppl 1):3-6

2 Sharma L, Cahue S, Song J, Hayes K, Pai YC, Dunlop D. Physical functioning over three years in knee osteoarthritis: role of psychosocial, local, mechanical, and neuromuscular factors. Arthitis Rheum 2003;48:3359-70.

3 Jette AM, Haley SM, Coster WJ, Kooyoomiian JT, Levenson S, Heeren T, et al. Late life function and disability instrument (FDI). J Gerontol A Biol Sci Med Sci 2002;57:M209-16.

4 Briggs A, Scott E, Steele K. Impact of osteoarthritis and analgesic treatment on quality of life of an elderly population. Ann Pharmacother 1999;33:1 154-9.

5 Hopman-Rock M, Kraaimaat FW, Biilsma JW. Quality of life in elderly subjects with pain in the hip or knee. Qual Life Res 1997;6:67-76.
6 Hill CL, Parsons J, Taylor A, Leach G. Health-related quality of life in a population sample with arthritis. J Rheumatol 1999;26:2029-35.

7 Wiklund I. Quality of life in arthritis patients using nonsteroidal antiinflammatory drugs. Can J Gastroenterol 1999;13:129-33.

8 de Bock GH, Kaptein AA, Touw-Otten F, Mulder JD. Health-related quality of life in patients with osteoarthritis in a family practice setting. Arthritis Care Res 1995;8:88-93.

9 Zhao SZ, Dedhiya SD, Bocanegra TS, Fort JG, Kuss ME, Rush SM. Healthrelated quality of life effects of oxaprozin and nabumetone in patients with osteoarthritis of the knee. Clin Ther 1999;21:205-17.

10 Yeomans N, Wilson I, Langstrom G, Hawkey C, Naesdal J, Walan A, et al. Quality of life in chronic NSAID users: a comparison of the effects of omeprazole and misoprostol. Scand J Rheumatol 2001;30:328-34.

11 Ware JE Jr, Sherbourne CD. The MOS 36-item Short-Form Health Status Survey (SF-36): conceptual framework and item selection. Med Care 1992;30:473-83.

12 Ware JE. The SF-36 Health Survey. In: Spilker B, ed. Quality of life and pharmacoeconomics in clinical trials. 2nd ed. Philadelphia, PA: LippincottRaven, 1996:337-45.

13 McHorney CA, Ware JE Jr, Lu JF, Sherbourne CD. The MOS 36-item ShortForm Health Survey (SF-36): III. Tests of data quality, scaling assumptions, and reliability across diverse patient groups. Med Care 1994;32:40-66.

14 Razavi D, Gandek B. Testing Dutch and French translations of the SF-36 Health Survey among Belgian angina patients. J Clin Epidemiol 1998;51:975-81

15 Leplege A, Ecosse E, Verdier A, Perneger TV. The French SF-36 Health Survey: translation, cultural adaptation and preliminary psychometric evaluation. J Clin Epidemiol 1998;51:1013-23.

16 Bellamy N, Buchanan WW, Goldsmith CH, Campbell J, Stitt LW. Validation of WOMAC: a health status instrument for measuring clinically important patient relevant outcomes to antirheumatic drug therapy in patients with osteoarthritis of the hip or knee. J Rheumatol 1988;15:1833-40.

17 Ethgen O, Tellier V, Ben Sedrine W, De Maessener J, Gosset C, Reginster J-Y. Health-related quality of life and cost ambulatory care in osteoporosis: how may such outcome measures be valuable information to health decision makers and payers? Bone 2003;32:718-24.

18 Pavelka K, Gatterova J, Olejarova M, Machacek S, Giacovelli G, Rovati LC. Glucosamine sulfate use and delay of progression of knee osteoarthritis: a 3-year, ramdomized, placebo-controlled, double-blind study. Arch Intern Med 2002;162:2113-23.

19 Schnitzer TJ. Means, responders, and meaning: evaluation of clinical trials in osteoarthritis. Arthritis Rheum 2003;48:3001-3.

20 McKenna F, Borenstein D, Wendt H, Wallemark C, Lefkowith JB, Geis GS. Celocoxib versus diclofenac in the management of osteoarthritis of the knee: a placebo-controlled, randomised, double-blind comparison. Scand J Rheumatol 2001;30:11-8

21 Yocum D, Fleischmann R, Dalgin P, Caldwell J, Hall D, Roszko P. Safety and efficacy of meloxicam in the treatment of osteoarthritis: a 12-week, double blind, multiple-dose, placebo-controlled trial. Arch Intern Med 2000; 160:2947-54.

22 Langman M, Kahler KH, Kong SX, Zhang Q, Finch E, Bentkover JD, et al. Drug switching patterns among patients taking non-steroidal antiinflammatory drugs: a retrospective cohort study of a general practitioners database in the United Kingdom. Pharmacoepidemiol Drug Safety 2001;10:517-24.

23 Bradley J D, Brandt KD, Katz BP, Kalasinski LA, Ryan SI. Comparison of an antiinflammatory dose of ibuprofen, an analgesic dose of ibuprofen, and acetaminophen in the treatment of patients with osteoarthritis of the knee. N Engl J Med 1991;325:87-91.

24 Lin J, Zhang W, Jones A, Doherty M. Efficacy of topical non-steroidal antiinflammatory drugs in the treatment of osteoarthritis: meta-analysis of randomised controlled trials. BMJ 2004;329:324-6.

25 La Montagna G, Tirri G, Cacace E, Perpignano G, Covelli M, Pipitone V, et al. Quality of life assessment during six months of NSAID treatment: Gonarthrosis and Quality Of Life (GOAL) study. Clin Exp Rheumatol 1998;16:49-54.

26 Samsa G, Edelman D, Rothman LM, Williams R, Lipscomb J, Matchar D. Determining clinically important differences in health status measures. A general approach with illustration to the health utilities index mark II. Pharmacoeconomics 1999;15:141-55.

27 Bindman AB, Keane D, Lurie N. Measuring health changes among severely ill patients. The floor phenomenon. Med Care 1990;28:1142-51.

28 Chambless LE, Roeback JR. Methods for assessing difference between groups in change when initial measurement is subject to intra-individual variation. Stat Med 1993;12:1213-37.

29 Bruyère $\mathrm{O}$, Honore A, Rovati LC, Giacovelli G, Henrotin YE, Seidel L, et al Radiologic features poorly predict clinical outcomes in knee osteoarthritis. Scand J Rheumatol 2002;31:13-16.

30 Rabenda V, Belaiche J, Raeman F, Haim M, Reginster J-Y. Use of healthcare resources related to gastrointestinal toxicity of NSAIDs [abstract]. Osteoporos Int 2003;14(suppl 7):S14. 